\title{
Analysis of Single Leg Squat and Single Leg Landing Performance Variability among Professional Soccer Players Across a Season
}

\author{
Msaad Alzhrani ${ }^{1{ }^{*},}$ Ahmad Alanazi ${ }^{1}$, Mazen Alqahtani ${ }^{1}$, Mohamed Sherif Sirajudeen ${ }^{1}$, \\ Lee Herrington ${ }^{2}$, Richard K. Jones ${ }^{3}$ \\ ${ }^{1}$ Department of Physical Therapy \& Health Rehabilitation, College of Applied Medical Sciences, \\ Majmaah University, Majmaah 11952, Saudi Arabia \\ ${ }^{2}$ Centre for Health Sciences Research, University of Salford, Salford, Manchester, UK \\ ${ }^{3}$ School of Health Science, University of Salford, Salford, UK
}

Received August 18, 2021; Revised November 9, 2021; Accepted November 22, 2021

\section{Cite This Paper in the following Citation Styles}

(a): [1] Msaad Alzhrani, Ahmad Alanazi, Mazen Alqahtani, Mohamed Sherif Sirajudeen, Lee Herrington, Richard K. Jones, "Analysis of Single Leg Squat and Single Leg Landing Performance Variability among Professional Soccer Players Across a Season," International Journal of Human Movement and Sports Sciences, Vol. 9, No. 6, pp. 1460 - 1469, 2021. DOI: 10.13189/saj.2021.090643.

(b): Msaad Alzhrani, Ahmad Alanazi, Mazen Alqahtani, Mohamed Sherif Sirajudeen, Lee Herrington, Richard K. Jones (2021). Analysis of Single Leg Squat and Single Leg Landing Performance Variability among Professional Soccer Players Across a Season. International Journal of Human Movement and Sports Sciences, 9(6), 1460 - 1469. DOI: 10.13189/saj.2021.090643.

Copyright $\mathrm{C} 2021$ by authors, all rights reserved. Authors agree that this article remains permanently open access under the terms of the Creative Commons Attribution License 4.0 International License

\begin{abstract}
Objectives: This study investigated the performance variability between limb type (dominant versus non-dominant leg) with regard to time (over season with three categories: pre-, start- and end-season) of professional soccer players. Methods: A total of 88 out of 124 male soccer players aged 16-30 years were asked to perform the specific athletic tasks single leg squat (SLS) and single leg landing (SLL) during different times in a full sports season. These functional tasks were evaluated using outcome measures of coefficient of variation, including frontal plane projection angle (FPPA) and hip adduction angle (HAA). Results: No significant difference was observed across all events between the dominant and non-dominant leg of the participants between screening sessions over time, for both SLS and SLL functional activities, except for one event in both second-order coefficient of variation for FFPA (FPPA V2) and HAA in SLS (at the start of season, $p=0.006$ ). The variations in FPPA and HA are clinically irrelevant, as no statistically significant differences were observed between limb types (dominant versus non-dominant leg) between screening sessions over a season, thereby suggesting that the performance variability is fairly consistent over time.
\end{abstract}

Conclusions: There is consistency across the sports season in the performance variability of SLS and SLL.

Keywords Anterior Cruciate Ligament, Knee Injury, Single Leg Squat, Single Leg Land, Frontal Plane Projection Angle, Performance Variability

\section{Introduction}

Among athletes, evaluation of biomechanical tasks is an important assessment tool and is commonly used not only for appraising performances, but also for rehabilitation on the field and in clinical practice [1-3]. These functional tasks can be used as important benchmarks to predict factors responsible for injuries [3]. Single leg squat (SLS) and single leg landing (SLL) are two important simple physical examination tests that are commonly utilized to identify abnormal frontal plane lower extremity kinematics, which could be associated with a large number of factors responsible for the lower limb injuries $[4,5]$. 
The present literature suggests that the highest incidence of Anterior cruciate ligament (ACL) injuries is observed among soccer players, followed by gymnasts, costing billions of dollars for treatment and rehabilitation and sometimes taking almost a year for the athlete to return to pre-injury levels [6]. Therefore, using SLS and SLL as early screening tools for individuals at higher risk of ligament injury is crucial, as they are found to be associated with classical abnormal kinematics, including increased hip medial rotation and adduction, and medial tibial rotation resulting in medial knee displacement known as dynamic knee valgus [4,7-12]. This suggests that while there are a number of studies to establish the performance of the tasks and their correlation to lower limb injuries, very few studies, to date, have examined the effects of performance variability on lower limb kinematics to explore their possible connection with lower limb injuries.

Variability in movements refers to the amount of movement change recorded between subsequent repetitions within an individual and has generated considerable interest among sports biomechanists to assess the role of such factors in causing injuries [13]. In a dynamic environment, no two movements are usually the same; therefore, the effect of variability in movements assessed in the form of comparisons among performance-variability has been evaluated in a few significant studies $[14,15]$. While both of these earlier studies evaluated the effects of movement variability among symptomatic versus asymptomatic patients, to the best of our knowledge, no studies have attempted to assess the kinematics of performance variability among athletes with respect to limb dominance over time. Hence the objective of the current study was to investigate the performance variability between limb type (dominant versus non-dominant leg) with regard to time (over season with three categories: pre-, start- and end-season) of professional soccer players.

\section{Methods}

\subsection{Participants}

A total of 124 male soccer players were taken from four professional local soccer clubs in Northwest of England. Players who consented to participate and met the inclusion criteria were included in the study. Professional soccer players in the age group of 16-30 years, who had suffered no major or significant injury in the lower limbs in the past six months and were able to perform single-leg squat and single-leg landing tasks independently without aids, and were willing to participate, were included in the study. A total of 90 players out of 124 agreed to be part of the study, with 88 players ultimately studied. The Research, Innovation and Academic Engagement Ethical Approval
Panel, University of Salford provided granted ethical approval for this study (HSCR15-27), where all the principles of Helsinki, 2013 were followed. All the participants signed a written informed consent prior to the participation in this study.

\subsection{Materials}

Evaluation of the two functional tasks (SLS and SLL) was performed using two digital cameras, called two-dimensional or 2D analysis, using Sony Handycam (Model No. DCR- HC37, Sony Corp, Tokyo, Japan) in accordance with the standard protocols performed in previous studies $[12,16,17]$. The devices were provided by the DSR, College of Applied Medical Sciences, Department of Physical Therapy at Majmaah University. Other tools used in the study included a 28 centimeter step and collaboration triangle.

\subsection{Procedure}

The participants were asked to perform specific athletic tasks, namely SLS and SLL five times each. For SLS, the subjects were asked to stand on one leg facing the camera and were instructed to squat down as low as possible. During SLS, the knee is flexed between $60^{\circ}$ to $80^{\circ}$ and held in that position for five seconds. A pilot study was conducted beforehand to train the therapists to record knee flexion using a standard goniometer. The therapists recording the measurements were blinded to avoid bias. The subjects were asked to initiate the movement with the first count, the second count was suggestive of the mid-path in SLS, the third indicated the lowest point of the squat, and the fifth indicated the end. This standardized the test for all participants, thereby reducing the effect of velocity on knee angles. Trials were only accepted if the subject squatted to the minimum desired degree of knee flexion while maintaining their balance throughout [17]. A total of five consecutive trials were performed on both legs individually to compare performance variability.

During SLL, the subjects were instructed to jump from a 28 centimeter step, leaning forward, and dropping as vertically as possible. They were asked to take a unilateral stance on the contralateral limb and then to step forward to drop onto the floor corresponding to the landing leg, ensuring that the contralateral leg makes no contact with any other surface, for five trials [18]. Both SLS and SLL were individually conducted on both legs (dominant and non-dominant leg).

\subsection{Measurements}

For each subject, knee frontal plane projection angle (FPPA) and hip adduction angle (HAA) data were recorded for both the dominant and non-dominant leg 
individually during the execution of SLS and SLL. The subjects were asked to perform five successful trials for each task and for each leg, and a successful trial required the movement to occur in the field of the 2D camera. The videotaping recorded the subject's trunk and lower limbs only. Sampling at $30 \mathrm{fps}$ was used. Standard methodology was used for recording the movements by placing the camera at a height of $60 \mathrm{~cm}$ from the floor, $2 \mathrm{~m}$ anterior to the subject's landing target, and was aligned perpendicular to the frontal plane [19]. Markers were placed at the midpoint of the ankle malleoli for the center of the ankle joint, the midpoint of the femoral condyles to approximate the center of the knee joint, and on both the anterior superior iliac spines (ASIS) [20]. The measurements for HA and knee FPPA for both tasks were derived using the Quintic Biomechanics software (version 26). FPPA is defined as the angle subtended between the lines from the markers on the ASIS to the knee joint and the line from the knee joint to the ankle. HAA is defined as the angle between the line from the marker on the ASIS to the knee joint, and the line connecting both ASISs. Both FPPA and HAA were measured at the frame, which represents the point of maximum knee flexion. This was determined as the lowest point of the squat and landing tasks. The negative FPPA means a valgus knee while a positive means a varus knee.

\subsection{Statistical Analysis}

The variability in performance was measured using the second-order coefficient of variation, referred to as V2 [21]. It is important to mention that the second-order coefficient (V2) accommodates values between 0 and 1 to make interpretations simple; a value of 0 represents no variability and a value of 1 is interpreted as the highest variability.

The IBM Statistical Package for the Social Sciences (SPSS) v23 was used to conduct all statistical tests. Two participants with missing data were excluded and the data of 88 participants were analyzed. The variability in performance with reference to limb type and time was evaluated with two-way repeated measures analysis of variance (ANOVA) using both FPPA V2 and HAA V2 for both the functional tasks for all 88 players $(n=88)$. The analysis of the standardized residuals using the ShapiroWilk test indicated that the variables were not distributed normally. It was observed that all the variables were normally distributed except start-season non-dominant SLS-HAA, pre-season dominant SLL-HAA, pre-season non-dominant SLL-HAA, and start-season non-dominant SLL-HAA.

Therefore, non-parametric tests were used for statistical evaluation, irrespective of the distribution patterns. It is important to note that the Friedman test, which is generally used for one-way repeated measures ANOVA, was utilized for statistical testing as no non-parametric alternative was available for measuring two-way repeated measures ANOVA, an alternative for a one-way repeated measures ANOVA. As such, the interaction between time and limb was not measured, and only the assessment for performance variability over time was carried out using the Friedman test.

Similarly, if variability over time was found to be statistically significant (using the Friedman test), then a comparison of differences between dominant and non-dominant limbs and between any two of the pre-, start- and end-season screening sessions would be evaluated using the Wilcoxon signed-rank test. If the Friedman test tested negative, no further tests were conducted. Likewise, when the assumption of the distribution shape of the differences between the two related groups was not symmetrical, the sign test was used instead of the Wilcoxon test.

\section{Results}

\subsection{Performance Variability Descriptive Statistics}

The data were not normally distributed; therefore, the descriptive statistics were represented as medians (Mdn) of the second-order coefficient of variation (V2) and interquartile range (IQR) for FPPA and HAA for both legs (dominant and non-dominant) for both tasks, for each screening session (pre-season, start of season, and end of season).

\subsection{Pre-season Performance Variability across Both Functional Tasks}

For the SLS activity, the dominant leg FPPA V2 $(\mathrm{n}=$ 88) had a median of 0.49 and IQR of 0.45 , whereas the non-dominant leg FPPA V2 median was 0.58 and IQR of 0.50 . Similarly, for the dominant leg HAA V2 $(n=88)$ had a median of 0.04 and IQR of 0.02 compared to the non-dominant leg where HAA V2 median was 0.03 and IQR of 0.03 , as represented in Fig. 1.

For the SLL activity, the dominant leg FPPA V2 median was 0.61 and IQR of 0.51 , whereas the non-dominant FPPA V2 median was 0.70 and IQR of 0.47 . Likewise, for the dominant leg, the HAA V2 median was 0.041 and IQR was 0.03 , whereas the median of non-dominant leg HAA V2 was 0.046 and IQR of 0.03 , as shown in Fig. 1.

\subsection{Start-Season Performance Variability}

For the SLS, as represented earlier, during the start of season the FPPA V2 for the dominant leg $(n=77)$ had a median of 0.49 and IQR of 0.48 , whereas the non-dominant FPPA V2 had a median of 0.62 and IQR of 0.51. Similarly, the dominant leg HAA V2 had a median 
of 0.04 and IQR of 0.03 , in comparison to the non-dominant leg HAA V2 where the median was 0.036 and IQR of 0.03 (Fig. 2).

For the SLL activity, the dominant limb FPPA V2 $(\mathrm{n}=$ 75 ) had a median of 0.65 and IQR of 0.44 , whereas the non-dominant FPPA V2 median was 0.76 and IQR of 0.46 . Likewise, the HAA V2 median for the dominant leg $(\mathrm{n}=$ 75) was 0.04 and IQR of 0.02 , compared to the non-dominant leg with an HAA V2 median of 0.04 and IQR of 0.03 (Fig. 2)

\section{Pre-season}

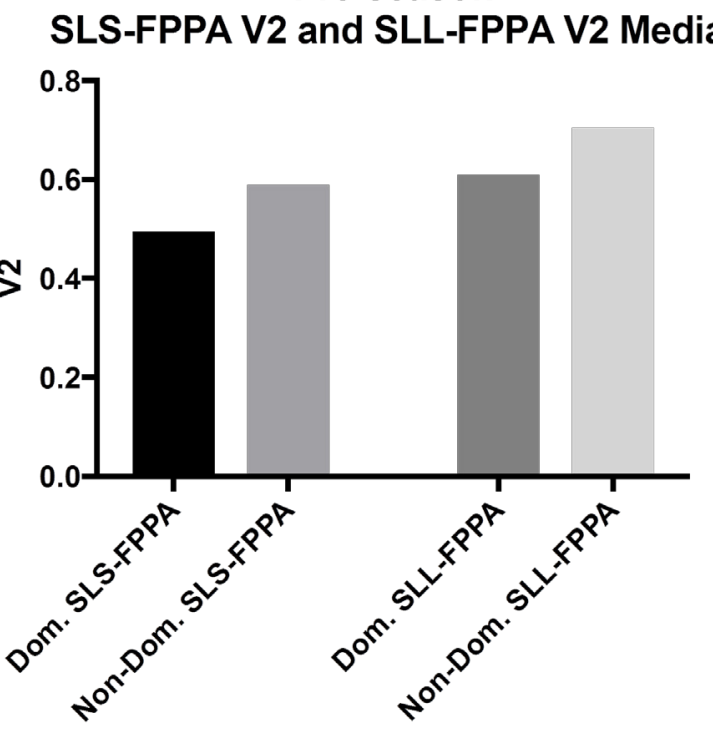

\section{Pre-season}

SLS-Hip angle V2 and SLL-Hip angle V2 Median

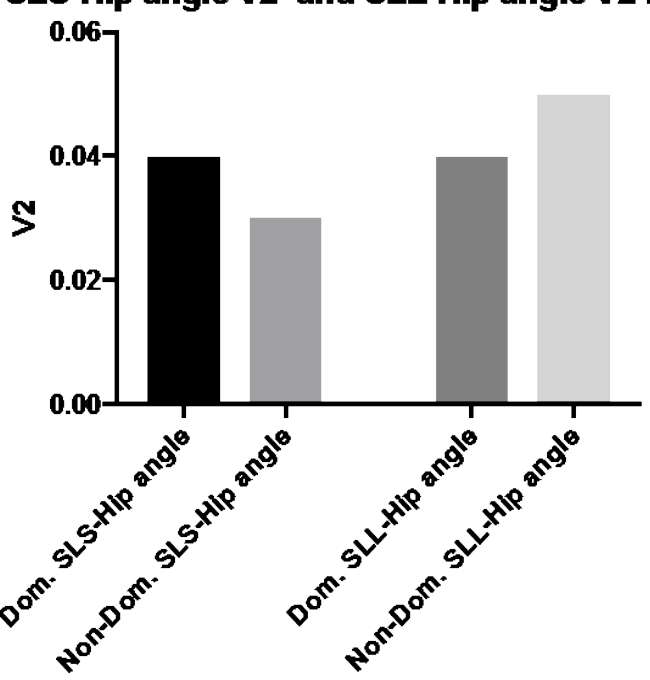

Dom - Dominant leg, FPPA - Frontal plane projection angle, HAA- Hip adduction angle, Non- Dom - Nondominant, SLL- Single leg landing, SLS Single leg squat, V2 - Second-order coefficient of variation

Figure 1. The median of pre-season performance variability (V2) for FPPA and HAA in both legs
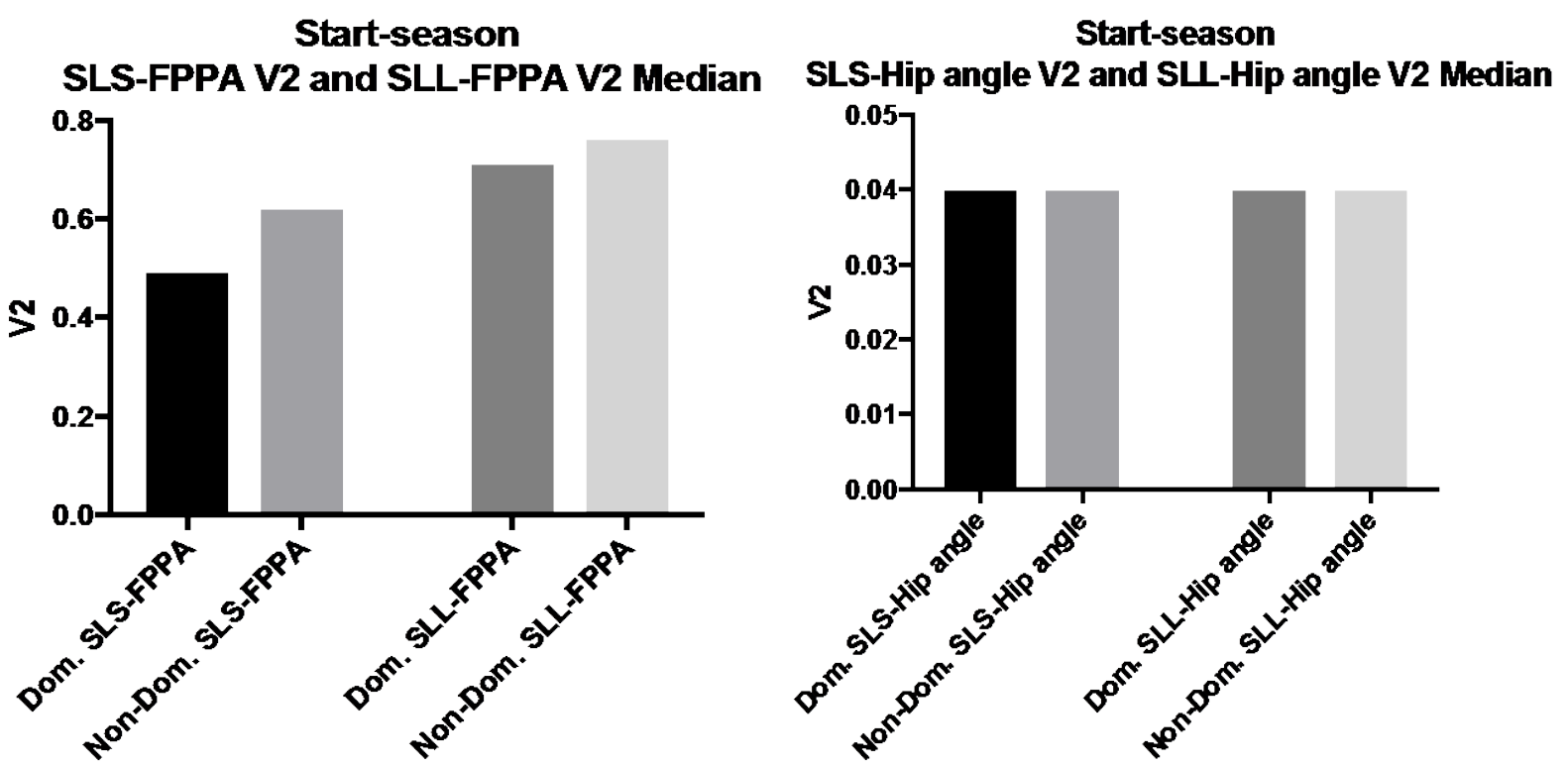

Dom - Dominant leg, FPPA - Frontal plane projection angle, HAA- Hip adduction angle, Non- Dom - Nondominant, SLL- Single leg landing, SLS Single leg squat, $\mathbf{V 2}$ - Second-order coefficient of variation

Figure 2. The median start-season performance variability (V2) for FPPA and HAA in both legs 


\subsection{End-Season Performance Variability}

Within the SLS activity, the FPPA V2 for the dominant leg $(n=67)$ had a median of 0.52 and IQR of 0.54 , whereas the non-dominant leg showed an FPPA V2 median of 0.49 and IQR of 0.64 . Similarly, the dominant leg HAA V2 $(n=67)$ had a median of 0.04 and IQR of 0.03 compared to HAA V2 for the non-dominant leg, where the median was 0.039 and IQR of 0.03 (Fig. 3).

In the SLL activity, the FPPA V2 median for the dominant leg $(\mathrm{n}=65)$ was 0.68 and IQR of 0.30 , compared to the FPPA V2 median for the non-dominant leg which was 0.73 and IQR of 0.45 . The HAA V2 for the dominant leg $(\mathrm{n}=65)$ had a median of 0.04 and $\mathrm{IQR}$ of 0.02 , whereas for the non-dominant leg HAA V2, the median was 0.040 and IQR was 0.03 (Fig. 3).

\subsection{Within-Session Variability in Performance (with reference to limb type)}

The investigation of variability in performance between the dominant and non-dominant limbs for each screening test was evaluated using sign tests of Wilcoxon signed-rank tests. To avoid type 1 error, the alpha level of $(\alpha=0.05)$ was adjusted to $(\alpha=0.05 / 3=0.016)$ for multiple comparisons.

\subsubsection{Pre-season}

For SLS, while evaluating for variability in performance with reference to limb types, Wilcoxon signed-rank test showed no significant difference both for FPPA as well as for HAA V2 ( $\mathrm{p}=0.196$ and $\mathrm{p}=0.076$, respectively). Likewise, for SLL, no statistically significant difference was noted between the limbs for both FPPA and HAA V2 $(\mathrm{Z}=1.58, \mathrm{p}=0.114$ and $\mathrm{Z}=$ $0.93, \mathrm{p}=0.0351$, respectively) (Table 1$)$.

\subsubsection{Start of season}

While evaluating for SLS, statistically significant differences were noted for dominant SLS-FPPA V2 (median $=0.49)$ as compared to the non-dominant counterpart SLS-FPPAV2 (median $=0.62$ ) at the start season screening $(Z=2.72, p=0.006$, median difference of -0.13 ), as represented in Table 1 . However, while evaluating for HAA (in the SLS activity), no difference was observed between the legs $(Z=-0.68, p=0.494)$. With reference to SLL evaluation, no statistically significant difference was observed between the limbs for both FPPA V2 $(\mathrm{Z}=1.18, \mathrm{p}=0.237)$ or HAA V2 $(\mathrm{Z}=$ $0.14, p=0.891)$.
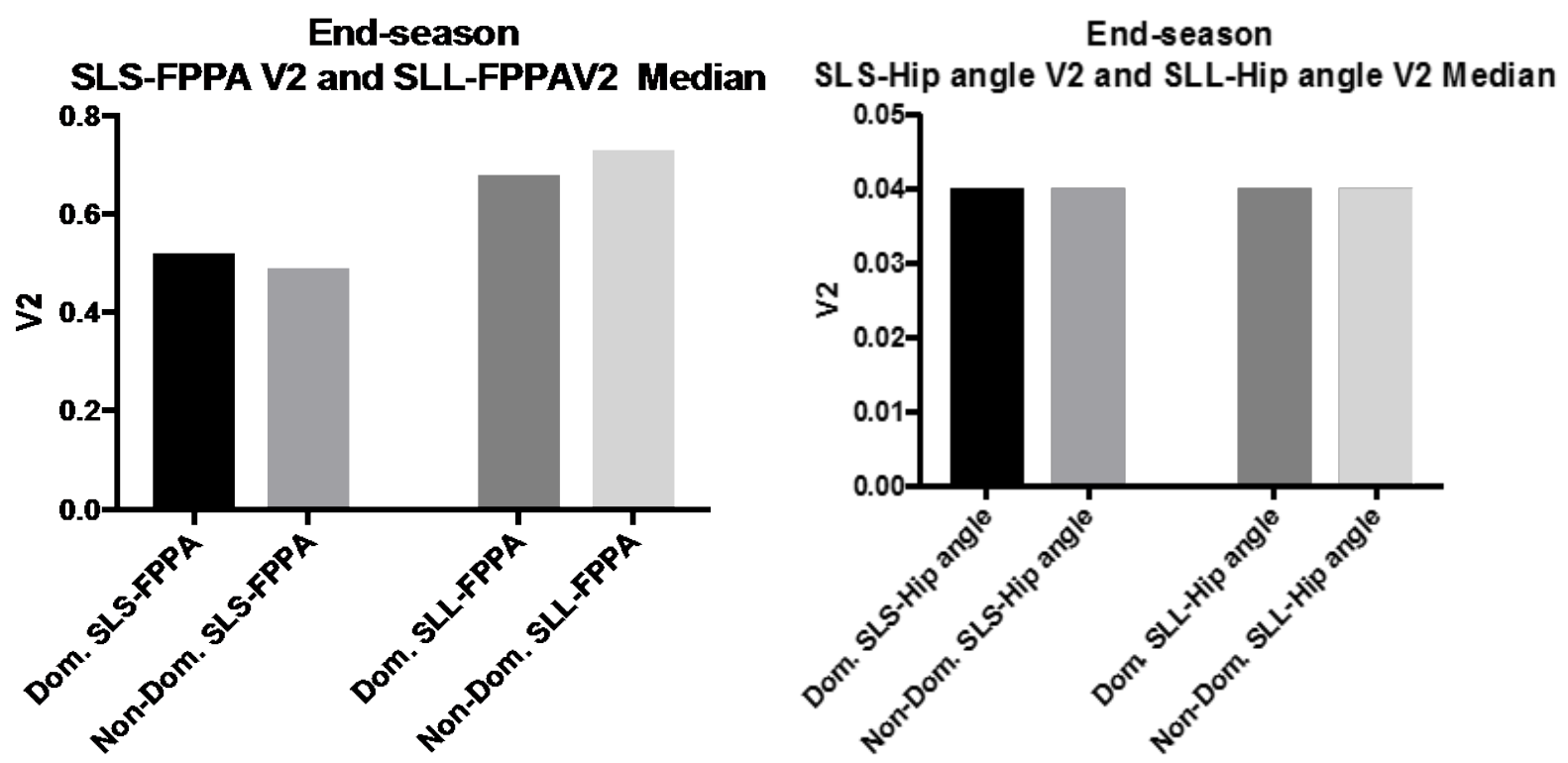

Dom - Dominant leg, FPPA - Frontal plane projection angle, HAA- Hip adduction angle, Non- Dom - Nondominant, SLL- Single leg landing, SLS Single leg squat, V2 - Second-order coefficient of variation

Figure 3. The median of end-season performance variability (V2) for FPPA and HAA in both legs 
Table 1. Results of statistical tests between legs for FPPA V2 and HAA V2 for both tasks

\begin{tabular}{|c|c|c|c|c|c|c|c|}
\hline \multicolumn{2}{|c|}{ Screening session } & & $\begin{array}{c}\text { Dom. leg } \\
\text { Median }\end{array}$ & $\begin{array}{c}\text { Non-Dom. leg } \\
\text { Median }\end{array}$ & Stat. test & $\begin{array}{c}\text { Median } \\
\text { difference }\end{array}$ & Pvalue \\
\hline \multirow{4}{*}{ Pre-season(a) } & \multirow{2}{*}{$S L S$} & FPPA V2 & 0.49 & 0.58 & $\mathrm{Z}=1.29$ & -0.09 & $\mathrm{p}=0.196$ \\
\hline & & HAA V2 & 0.044 & 0.035 & $Z=-1.78$ & -0.031 & $\mathrm{p}=0.076$ \\
\hline & \multirow{2}{*}{$S L L$} & FPPA V2 & 0.61 & 0.70 & $Z=1.58$ & -0.09 & $\mathrm{p}=0.114$ \\
\hline & & HAA V2 & 0.041 & 0.046 & $\mathrm{Z}=0.93$ & -0.005 & $\mathrm{p}=0.351$ \\
\hline \multirow{4}{*}{ Start-season(b) } & \multirow{2}{*}{$S L S$} & FPPA V2 & 0.49 & 0.62 & $Z=2.72$ & -0.13 & $\mathrm{p}=0.006^{*}$ \\
\hline & & HAA V2 & 0.040 & 0.036 & $Z=-0.68$ & 0.004 & $\mathrm{p}=0.494$ \\
\hline & \multirow{2}{*}{$S L L$} & FPPA V2 & 0.65 & 0.76 & $Z=1.18$ & -0.11 & $\mathrm{p}=0.237$ \\
\hline & & HAA V2 & 0.045 & 0.043 & $\mathrm{Z}=0.14$ & 0.002 & $\mathrm{p}=0.891$ \\
\hline \multirow{4}{*}{ End-season(c) } & \multirow{2}{*}{$S L S$} & FPPA V2 & 0.52 & 0.49 & $Z=0.28$ & 0.03 & $\mathrm{p}=0.779$ \\
\hline & & HAA V2 & 0.040 & 0.039 & $\mathrm{Z}=0.32$ & 0.001 & $\mathrm{p}=0.750$ \\
\hline & \multirow[b]{2}{*}{$S L L$} & FPPA V2 & 0.68 & 0.73 & $Z=0.25$ & -0.05 & $\mathrm{p}=0.806$ \\
\hline & & $\begin{array}{c}\text { Hip angle } \\
\text { V2 }\end{array}$ & 0.038 & 0.040 & $Z=0.94$ & -0.002 & $\mathrm{p}=0.348$ \\
\hline
\end{tabular}

Dom - Dominant leg, FPPA - Frontal plane projection angle, HAA- Hip adduction angle Non- Dom - Nondominant, SLL- Single leg landing, SLS - Single leg squat, V2 - Second-order coefficient of variation, ${ }^{*}-\mathrm{p}<0.05$ (Significant)

Table 2. Performance variability change (FPPA V2 and HAA V2) for each leg over time using the Friedman test for both SLS and SLL

\begin{tabular}{|c|c|c|c|c|c|}
\hline \multicolumn{2}{|c|}{ Screening } & Leg & Variable & Stat. tests & $P$ value \\
\hline \multirow{8}{*}{$\begin{array}{c}\text { Between } \\
\text { sessions } \\
\text { (Over time) }\end{array}$} & \multirow{4}{*}{ SLS } & \multirow{2}{*}{ Dom. } & FPPA V2 & $\chi 2(2)=4.03$ & $\mathrm{p}=0.133$ \\
\hline & & & HAAV2 & $\chi 2(2)=.984$ & $\mathrm{p}=0.611$ \\
\hline & & \multirow{2}{*}{ Non-dom. } & FPPA V2 & $\chi 2(2)=2.89$ & $\mathrm{p}=0.236$ \\
\hline & & & HAA V2 & $\chi 2(2)=1.18$ & $\mathrm{p}=0.556$ \\
\hline & \multirow{4}{*}{ SLL } & \multirow{2}{*}{ Dom. } & FPPA V2 & $\chi^{2}(2)=1.60$ & $\mathrm{p}=0.449$ \\
\hline & & & HAA V2 & $\chi 2(2)=1.20$ & $\mathrm{p}=0.549$ \\
\hline & & \multirow{2}{*}{ Non-dom. } & FPPA V2 & $\chi 2(2)=2.43$ & $\mathrm{p}=0.296$ \\
\hline & & & HAA V2 & $\chi 2(2)=2.63$ & $\mathrm{p}=0.268$ \\
\hline
\end{tabular}

Dom - Dominant leg, HA - Hip angle, FPPA - Frontal plane projection angle, HAA- Hip adduction angle, Non- Dom - Nondominant, SLLSingle leg landing, SLS - Single leg squat, V2 - Second-order coefficient of variation,

\subsubsection{End-season}

Similar to other time points, no differences were observed for the SLS activity in FPPA $(\mathrm{Z}=0.28, \mathrm{p}=$ $0.779)$ and HAA $(Z=0.32, p=0.750)$, or the SLL activity in FPPA $(\mathrm{Z}=0.25, \mathrm{p}=0.806)$ and HAA $(\mathrm{Z}=0.49, \mathrm{p}=$ 0.348 ), as represented in Table 1.

In summary, performance variability of both FPPA and HAA in the dominant and non-dominant leg in the pre-, start-, and end-season screening sessions showed no significant statistical differences in either SLS or SLL. The only noted difference in tests was evident in the SLS activity for the FPPA measurement at the start-season screening, where the non-dominant leg showed the greatest performance variability.

\subsection{Between-Session Variability in Performance (variability differences over time)}

Figure 4 shows the change in performance for both the dominant and non-dominant leg over time (between sessions) for both FPPA V2 and HAA V2 for the functional tasks of both SLS and SLL.

While evaluating performance variability change for limb types (dominant and non-dominant) over time across a full season, no statistically significant differences were observed for both FPPA V2 and HAA V2 across the two screening sessions. Moreover, as no significant difference was observed over time for all variables in both SLS and SLL, there was no requirement to assess the between-two-sessions variable changes (Table 2).

Therefore, it can be summarized that no changes in performance variability were observed and both FPPA and HAA remained consistent over time (across a full season) for both the SLS $(p=0.13)$ and SLL tasks $(p=$ $0.61)$. 

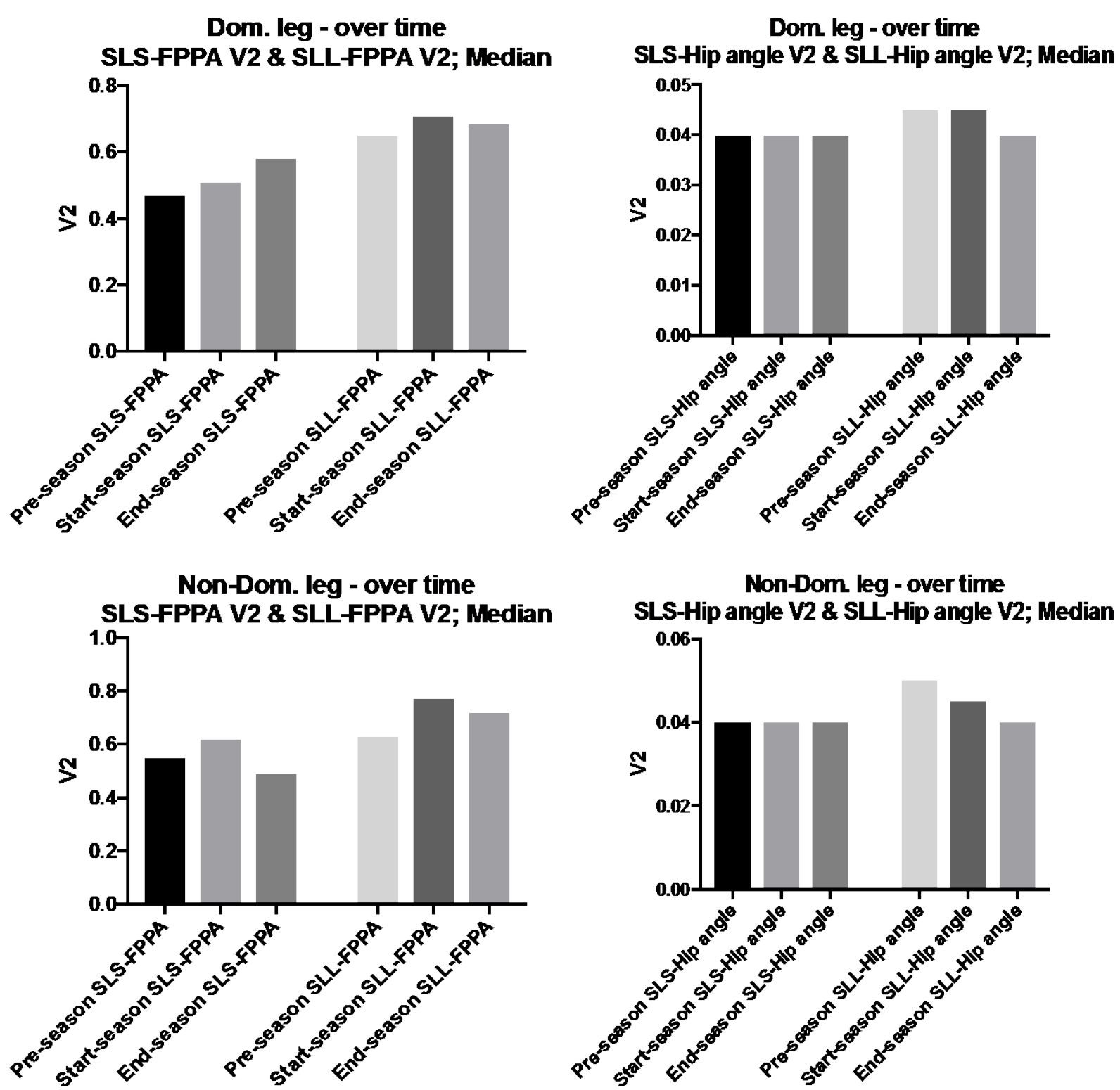

Dom - Dominant leg, FPPA - Frontal plane projection angle, HAA- Hip adduction angle, Non- Dom - Nondominant SLL- Single leg landing, SLS Single leg squat, $\mathbf{V} \mathbf{2}$ - Second-order coefficient of variation

Figure 4. Performance variability of the both legs over time (between sessions) with reference to FPPA V2 and HAA V2

\section{Discussion}

This study aimed to investigate the performance variability differences between limb type (dominant versus non-dominant leg) and with regard to time (over a full season with three categories: pre-, start-, and end-season) in professional soccer players. Players were asked to perform the functional tasks of SLS and SLL, evaluated using outcome measures of FPPA variability and HAA variability.

The study inferred that no significant difference was observed across all events between the dominant and non-dominant leg of the participants between screening sessions over time, in both SLS and SLL activities, except for one event where a significant difference was observed in both FPPA V2 measures for the SLS activity at the start of the season $(p=0.006)$ (Table 1).

In previously reported studies, the variability in performance was assessed while using different functional tasks, including SLL, running, side-step cutting, and single leg jump-landing [14,15,22-26]. While Brown et al. [14] used SLL to evaluate variability in movements in patients with chronic ankle injury, Pollard et al. [15] used the functional task of side-step cutting among soccer players after ACL reconstruction to evaluate changes in movements. This was studied in more detail by evaluating load accommodation strategies as well as movement variability in a single functional task of SLL [23]. In another study by Scholtes and Salsich, SLS was evaluated for assessing movement variability among patients with 
patella-femoral pain [24]. In yet another series of studies, Fox et al. [25] used the functional tasks of SLS and SLL to evaluate injuries among netball players, while more recently Mail et al. [26] used SLL to evaluate the movement variability with reference to FPPA among a wide range of athletes including cyclists, runners, and squash players. However, all of the studies mentioned were either cross-sectional in nature and evaluated over a particular period, or performed with reference to post-injury assessment.

The present study varying from earlier studies was conducted, as it attempted to evaluate the variability in performance with regard to limb type (dominant versus non-dominant) among a common group of subjects, that is, professional soccer players over a full season (pre, start, and end of season), which has, to date, not been conducted.

Another characteristic difference in the present study in relation to earlier studies is that previous researches have always relied upon variables such as step width, step length, stance time, swing time, jump height, and foot placement to assess performance variability, with no recorded variable of measurement usage of joint kinematics.

No marked difference was observed in performance variability between the dominant and non-dominant legs in the current study, other than the FPPA V2 of the SLS activity at the start-season screening $(\mathrm{p}<0.006)$, although this was not greater than the standard error of measurement i.e, $1.96^{\circ}$ and $1.99^{\circ}$ for SLS and SLL respectively. During SLS at the start of season, the difference between legs was $0.45^{\circ}$ (calculated using the average difference of FPPA V2 between legs, which was approximately $13 \%$ of $3.52^{\circ}$ ). The value was smaller than the standard error of measurement, which is $1.41^{\circ}$ in SLS, indicating that in both SLL and SLS activities, FPPA and HAA between legs indicated no significant difference in performance variability. Although more performance variability was observed in FPPA in SLL than in SLS, this could be attributed to the fact that SLL is a complex and dynamic functional task as compared to SLS [27-31]. Overall, no significant difference was observed between sessions of FPPA and HAA, while evaluating both functional tasks of SLS as well as SLL, suggesting no performance variability in terms of limb variation (dominant versus non-dominant) across the season. The novel observations in the present study are, to the best of our knowledge, the first of its kind to be reported. Therefore, for all practical purposes, the variations in FPPA and HAA are clinically irrelevant, as no statistically significant differences were observed between limb types (dominant versus non-dominant leg) between screening sessions over a season, thereby suggesting that the performance variability is fairly consistent over time.

This research does have some limitations. The present study used a second-order coefficient of variation (V2) to evaluate performance variability. This component is thought to be less sensitive than the principal component analysis (PCA), which is used for $3 \mathrm{D}$ analysis $[32,13]$. Nevertheless, V2 remained the best option available in comparison to the other regular coefficient of variations and was used quite effectively to draw valuable inferences [21]. Similarly, the number of repetitions made (five trials each for SLS as well as SLL) lacks scientific rationality; determining the optimal number of trials for clinical significance is a difficult task to evaluate for a variety of reasons $[13,33]$. To calculate the correct quantity of repetitions needed to obtain a stable mean, deductive analysis using sequential estimation must be used since the successive mean variation is within a range that approximates the overall average [13]. Future research should focus on keeping the lacunae in focus, with more attention being paid towards investigating the relationship between lower limb performance-variability and non-contact injuries as well as using the better modes of evaluating the biomechanical tasks.

\section{Conclusion}

The results of this study emphasize the fact that no significant differences were found in performance variability between dominant and non-dominant legs in either SLS or SLL. Furthermore, this study demonstrated that there is consistency across the sports season in the performance variability of SLS and SLL. Further studies could be designed in the future to establish the relationship between performance variability and lower limb injuries.

\section{Acknowledgment}

The authors are grateful to the soccer players who participated in this study.

\section{Declarations}

\section{Conflicts of interest}

We affirm that we have no financial affiliation or involvement with any commercial organization that has a direct financial interest in any matter included in this manuscript.

\section{Ethics approval}

The Research, Innovation and Academic Engagement Ethical Approval Panel, University of Salford provided granted ethical approval for this study (HSCR15-27).

\section{Consent to participate}

The Research, Innovation and Academic Engagement Ethical Approval Panel, University of Salford provided 
granted ethical approval for this study (HSCR15-27). All the participants signed a written informed consent prior to the participation in this study.

\section{REFERENCES}

[1] Delextrat A, Cohen D. Physiological testing of basketball players: toward a standard evaluation of anaerobic fitness. J Strength Cond Res. 2008;22(4):1066-72. https ://doi.org/1 $0.1519 /$ JSC.0b013e3181739d9b.

[2] Hewett TE, Myer GD, Ford KR. Decrease in neuromuscular control about the knee with maturation in female athletes. J Bone Joint Surg Br. 2004;86(8):1601-8.

[3] Hughes T, Sergeant JC, van der Windt DA, et al. Periodic health examination and injury prediction in professional football (Soccer): theoretically, the prognosis is good. Sports Med. 2018;48(11):2443-8. https ://doi.org/ 10.1007/s40279-018-0928-y

[4] Willson JD, Davis IS. Utility of the frontal plane projection angle in females with patellofemoral pain. J Orthop Sports Phys Ther. 2008;38(10):606-15.https ://doi.org/10.2519/jo spt.2008.2706

[5] Whatman C, Hing W, Hume P. Kinematics during lower extremity functional screening tests-are they reliable and related to jogging?. Phys Ther Sport. 2011;12(1):22-9. https ://doi.org/10.1016/j.ptsp.2010.10.006

[6] Dodson CC, Secrist ES, Bhat SB, et al. Anterior cruciate ligament injuries in National Football League athletes from 2010 to 2013: a descriptive epidemiology study. Orthop J Sports Med. 2016;4(3):2325967116631949. https://doi.org /10.1177/2325967116631949.

[7] Hewett TE, Myer GD, Ford KR, et al. Biomechanical measures of neuromuscular control and valgus loading of the knee predict anterior cruciate ligament injury risk in female athletes: a prospective study. Am J Sports Med. 2005;33(4):492-501.

https://doi.org/10.1177/0363546504269591.

[8] Myer GD, Ford KR, Foss KD, et al. The incidence and potential pathomechanics of patellofemoral pain in female athletes. Clin Biomech. 2010;25(7):700-7. https://doi.org/1 0.1016/j.clinbiomech.2010.04.001

[9] Koga H, Bahr R, Myklebust G, et al. Estimating anterior tibial translation from model-based image-matching of a noncontact anterior cruciate ligament injury in professional football: a case report. Clin. J. Sport Med. 2011;21(3):271-4. https://doi.org/10.1097/JSM.0b013e31821899ec

[10] Waldén M, Krosshaug T, Bjørneboe J, et al. Three distinct mechanisms predominate in non-contact anterior cruciate ligament injuries in male professional football players: a systematic video analysis of 39 cases. Br. J. Sports Med. 2015;49(22):1452-60.

https://doi.org/10.1136/bjsports-2014-094573

[11] Petersen W, Rembitzki I, Liebau C. Patellofemoral pain in athletes. Open Access J. Sports Med. 2017;8:143. https://doi.org/10.2147/OAJSM.S133406
[12] Hughes T, Jones RK, Starbuck C, et al. Are tibial angles measured with inertial sensors useful surrogates for frontal plane projection angles measured using 2-dimensional video analysis during single leg squat tasks? A reliability and agreement study in elite football (soccer) players. J Electromyogr Kinesiol. 2019;44:21-30. https://doi.org/10.1 016/j.jelekin.2018.11.005

[13] Preatoni E, Hamill J, Harrison AJ, et al. Movement variability and skills monitoring in sports. Sports Biomech. 2013;12(2):69-92.

https://doi.org/10.1080/14763141.2012.738700

[14] Brown C, Bowser B, Simpson KJ. Movement variability during single leg jump landings in individuals with and without chronic ankle instability. Clin Biomech. 2012;27(1):52-63.

https://doi.org/10.1016/j.clinbiomech.2011.07.012

[15] Pollard CD, Stearns KM, Hayes AT, et al. Altered lower extremity movement variability in female soccer players during side-step cutting after anterior cruciate ligament reconstruction. Am J Sports Med. 2015;43(2):460-5 https://doi.org/10.1177/0363546514560153

[16] McLean SG, Walker K, Ford KR, et al. Evaluation of a two dimensional analysis method as a screening and evaluation tool for anterior cruciate ligament injury. Br J Sports Med. 2005;39(6):355-62.

https://doi.org/10.1136/bjsm.2005.018598

[17] Mizner RL, Chmielewski TL, Toepke JJ, et al. Comparison of two-dimensional measurement techniques for predicting knee angle and moment during a drop vertical jump. Clin J Sport Med. 2012; 22(3):221.https://doi.org/10.1097/JSM.0 b013e31823a46ce

[18] Munro A, Herrington L, Carolan M. Reliability of 2-dimensional video assessment of frontal-plane dynamic knee valgus during common athletic screening tasks. J Sport Rehabil. 2012;21(1):7-11.https://doi.org/10.1123/jsr. 21.1 .7

[19] Sorenson B, Kernozek TW, Willson JD, et al. Two-and three-dimensional relationships between knee and hip kinematic motion analysis: single-leg drop-jump landings. J Sport Rehabil. 2015;24(4):363-72. https://doi.org/10.1123/ jsr.2014-0206

[20] Herrington L, Alenezi F, Alzhrani M, et al. The reliability and criterion validity of 2D video assessment of single leg squat and hop landing. J Electromyogr Kinesiol. 2017;34:80-5.

https://doi.org/10.1016/j.jelekin.2017.04.004

[21] Kvålseth TO. Coefficient of variation: the second-order alternative. J Appl Statistics. 2017;44(3):402-15. https://doi.org/10.1080/02664763.2016.1174195

[22] Arshi AR, Mehdizadeh S, Davids K. Quantifying foot placement variability and dynamic stability of movement to assess control mechanisms during forward and lateral running. J Biomech. 2015;48(15):4020-5. https://doi.org/1 0.1016/j.jbiomech.2015.09.046

[23] Nordin AD, Dufek JS. Load accommodation strategies and movement variability in single-leg landing. J Appl Biomech. 2017;33(4):241-7. https://doi.org/10.1123/jab.2016-0097

[24] Scholtes SA, Salsich GB. A dynamic valgus index that 
combines hip and knee angles: assessment of utility in females with patellofemoral pain. Int. J. Sports Phys. Ther. 2017;12(3):333.

[25] Fox AS, Bonacci J, Saunders N. The relationship between performance of a single-leg squat and leap landing task: moving towards a netball-specific anterior cruciate ligament (ACL) injury risk screening method. Sports Biomech. 2018;19(4):1-7.https://doi.org/10.1080/1476314 1.2018.1498535

[26] Mail MS, Azhar NM, Affandi NF, et al. Relationship between isokinetic leg strength and knee frontal plane projection angle during single leg squat among male junior athletes. J Heal Transl Med. 2019;22(2):43-8. https://doi.org/10.22452/jummec.vol22no2.7

[27] Willson JD, Ireland ML, Davis I. Core strength and lower extremity alignment during single leg squats. Med Sci Sports Exerc. 2006;38(5):945-52. https://doi.org/10.1249/01.mss.0000218140.05074.fa

[28] Cortes N, Onate J, Abrantes J, et al. Effects of gender and foot-landing techniques on lower extremity kinematics during drop-jump landings. J Appl Biomech. 2007;23(4):289-99. https://doi.org/10.1123/jab.23.4.289
[29] Blackburn JT, Padua DA. Sagittal-plane trunk position, landing forces, and quadriceps electromyographic activity. J Athl Train. 2009;44(2):174-9. https://doi.org/10.4085/10 62-6050-44.2.174

[30] Donohue MR, Ellis SM, Heinbaugh EM, et al. Differences and correlations in knee and hip mechanics during single-leg landing, single-leg squat, double-leg landing, and double-leg squat tasks. Res Sports Med. 2015;23(4):394-411.

https://doi.org/10.1080/15438627.2015.1076413

[31] Räisänen AM, Pasanen K, Krosshaug T, et al. Association between frontal plane knee control and lower extremity injuries: a prospective study on young team sport athletes. BMJ Open Sport Exerc Med. 2018;4(1) https://doi.org/10.1136/bmjsem-2017-000311

[32] Muniz AM, Nadal J. Application of principal component analysis in vertical ground reaction force to discriminate normal and abnormal gait. Gait posture. 2009;29(1):31-5. https://doi.org/10.1016/j.gaitpost.2008.05.015

[33] Paz GA, de Freitas Maia M, Gualter H. Knee Frontal Plane Projection Angle: A Comparison Study Between Drop. J Sport Rehabil.2017;28(2):153-158.https://doi.org/10.1123/ jsr.2017-0204 